\title{
Cigar and pipe smoking related to four year survival of coronary patients
}

\author{
NOEL HICKEY, RISTEARD MULCAHY, LESLIE DALY, IAN GRAHAM, \\ SHEERA O'DONOGHUE, CAROL KENNEDY \\ From the Cardiac Department and Department of Preventive Cardiology and Department of Community Medicine \\ and Epidemiology, St Vincent's Hospital and University College, Dublin, Republic of Ireland
}

SUMMARY Six hundred and thirty-four male patients under 60 years who survived a first attack of unstable angina or myocardial infarction were followed for a period of four years. Details of initial and follow-up smoking habits were examined.

Patients who continued to smoke cigarettes or cigars had an excess mortality compared with non-smokers, with those who stopped smoking, and with cigarette smokers who changed to pipe smoking. Pipe smokers who continued smoking the pipe had an observed mortality which was greater than that of the non-smokers, but the numbers were small and the results were not statistically significant. The effect of smoking habit on mortality was not influenced by two other determinants of prognosis: age and severity of initial attack.

These results confirm that the long-term prognosis of patients after unstable angina or myocardial infarction may be significantly influenced by smoking habits. They are consistent with the hypothesis that cigar and pipe smoking may have an adverse effect after myocardial infarction but further studies are needed to corroborate the association between cigar and pipe smoking and prognosis of coronary heart disease.

A significantly lower long term mortality has been reported among survivors of myocardial infarction who stop smoking cigarettes compared with those who continue to smoke. ${ }^{1-5}$

While results from prospective epidemiological studies suggest that pipe and cigar smoking are less harmful than cigarette smoking, ${ }^{6}$ the effect of pipe and cigars on prognosis in coronary patients has not been reported. Cigarette smokers who change to pipe or cigars after myocardial infarction may differ in prognosis compared with those who stop all forms of smoking or those who are primary pipe or cigar smokers only.

\section{Subjects and methods}

The long term follow-up study of coronary heart disease at St Vincent's Hospital has been described elsewhere. ${ }^{13}$ Entry criteria for patients are: admission to the cardiac department with a first attack of acute myocardial infarction or unstable angina (rest pain and serial electrocardiographic changes), age less than

Accepted for publication 8 February 1983
60 years at admission, male sex, and survival for at least 28 days. Between January 1961 and December 1975, 664 subjects entered the study.

The present report concerns $638(96 \cdot 1 \%)$ men who survived for at least six months from the date of admission to the hospital. Six months survival was chosen to isolate the effect of smoking on survivors from that of infarct size which strongly influences prognosis in the early months. ${ }^{2}$ The smoking habits at entry of the 26 men who died between one and six months did not differ significantly from those of the 638 men who survived six months.

Of the 638 men included in the study, four had inadequate follow-up details of smoking habits and were excluded from analysis. None of the four died during the follow-up period. Seventy four $(11.7 \%)$ of the remaining 634 patients died over the four years from the date of admission to hospital.

The mean age of the patients at the time of admission to hospital was 51 years with an age range of 26 to 59. There were no significant differences in the mean ages in groups defined by initial and follow-up smoking categories.

Initial measurements included details of cigarette, 
pipe, and cigar smoking habits before the coronary attack. Current cigarette smoking was recorded as average number of cigarettes per day. Pipe smoking was recorded as ounces of tobacco per week. Cigar smoking was recorded in those who smoked one cigar per day or more but the number, size, or type of cigar were not specified. Inhalation was recorded for cigarette smokers only.

Follow-up information on smoking habits was recorded at three monthly intervals over the first year after discharge from hospital and thereafter at annual intervals. Details of smoking at follow-up included whether patients had stopped or continued smoking. If continuing to smoke, the number of cigarettes smoked was recorded. No details of the quantity was elicited from those smoking pipe or cigars.

Individuals were classified as non-smokers, cigarette, pipe, cigar, and mixed smokers both initially and at the examination closest to four year follow-up.

The $\chi^{2}$ test was used to assess statistical significance of the contrasts. Because of small numbers in many. categories some large and interesting differences failed to reach a probability level of less than $5 \%$. The method prescribed by Armitage ${ }^{7}$ was used to partition the $\chi^{2}$ value into orthogonal components to assess which factors may have contributed to a significant effect in tables larger than $2 \times 2$.

\section{Results}

Table 1 shows the initial and last follow-up smoking status of the 634 patients. It can be seen that the proportion of smokers declined from $82 \%$ to $53 \%$ through the follow-up period, with a corresponding rise in non-smokers from $18 \%$ to $47 \%$. Table 2 relates mortality to follow-up smoking habits. A mortality gradient may be observed, with cigar smokers experiencing the highest mortality followed by cigarette, pipe, and non-smokers $\left(\chi^{2}=9.95, \mathrm{df}=4\right.$, $p<0.05$ ). When the influence of the different forms of follow-up smoking on mortality is examined separately by partitioning the $\chi^{2}$ into independent components, it can be shown that the experience of the cigar smokers is not significantly different from that of cigarette or pipe smokers but that these three groups combined have a significantly higher mortality than the non-smokers.

Thirty-seven patients were classified as mixed smokers. These smoked more than one form of tobacco. Thirty-five of the mixed smokers were pipe smokers. Twenty-seven were cigarette smokers and there were 12 cigar smokers. This group included a number of intermittent and erratic smokers who were difficult to classify in any specific smoking group. Only one $(2.7 \%)$ of this group died over the four
Table 1 Initial and last follow-up smoking status of 634 patients with myocardial infarction

\begin{tabular}{llllll}
\hline Smoking status & \multicolumn{2}{l}{ Initial } & & \multicolumn{2}{l}{ Four years } \\
\cline { 2 - 3 } \cline { 5 - 6 } & No & $\%$ & & No & $\%$ \\
\hline Smoking & 520 & 82.0 & & 335 & 52.8 \\
Non-smoking & 114 & 18.0 & & 299 & 47.2 \\
Total & 634 & $100 \cdot 0$ & 634 & $100 \cdot 0$ \\
\hline
\end{tabular}

Table 2 Smoking habits at last follow-up and four year mortality

\begin{tabular}{lccc}
\hline $\begin{array}{l}\text { Smoking habit at last } \\
\text { follow-up }\end{array}$ & No. & \multicolumn{2}{c}{ Mortality } \\
\cline { 3 - 4 } & & No. & $\%$ \\
\hline Cigarettes & 196 & 30 & $15 \cdot 3$ \\
Cigars & 21 & 5 & 23.8 \\
Pipe & 81 & 10 & 12.3 \\
Mixed & 37 & 1 & 2.7 \\
None & 299 & 28 & 9.4 \\
Total & 634 & 74 & 11.7 \\
\hline
\end{tabular}

$\chi^{2}=9.95, \mathrm{df}=4, \mathrm{p}<0.05$

Table 3 Smoking status at last follow-up and four year mortality with mixed smokers allotted to individual smoking categories

\begin{tabular}{lccc}
\hline $\begin{array}{l}\text { Smoking habit } \\
\text { at last follow-up }\end{array}$ & No. & \multicolumn{2}{l}{ Mortality } \\
\cline { 3 - 4 } & & No. & $\%$ \\
\hline Cigarettes & 223 & 31 & 13.9 \\
Cigars & 33 & 5 & 15.1 \\
Pipe & 116 & 11 & 9.4 \\
Non-smokers & 299 & 28 & 9.4 \\
\hline
\end{tabular}

Note: One decedent is included in both cigarette and pipe groups.

years. Including these 37 in single smoking categories reduces the mortality gradient (Table 3 ). Cigar smokers had a mortality of $15 \cdot 1 \%$. Cigarette smokers had a mortality of $13.9 \%$. Pipe and non-smokers had a similar mortality of $9.4 \%$.

The choice of patients who survived their initial myocardial infarction by at least six months appears to have eliminated the effect of the severity of the initial attack on survival. Univariate analysis failed to show any differences in mortality in the different follow-up smoking groups according to the severity of the initial attack.

The effect of changing smoking status on mortality is shown in Table 4. Cigarette smokers at follow-up examination had a significantly higher mortality than non-smokers $\left(\chi^{2}=4.04, \mathrm{df}=1, \mathrm{p}<0.05\right)$. The higher mortality among cigarette smokers compared with pipe smokers during follow-up was not significant. Initial cigarette smokers who had stopped had a nonsignificant reduction in mortality compared with cigarette smokers who continued. Cigarette smokers 
Table 4 Observed mortality in different follow-up smoking groups

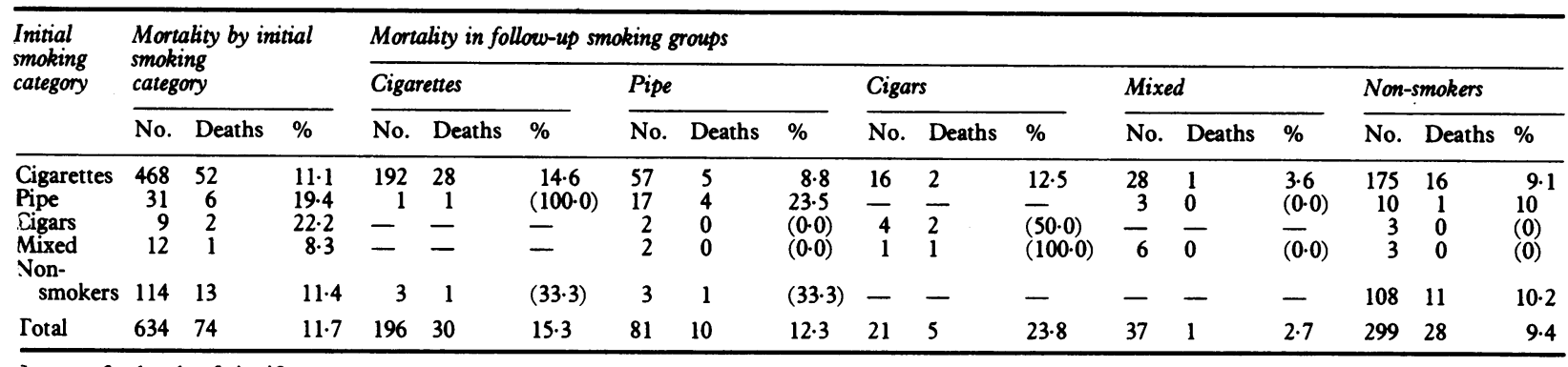

jee text for levels of significance.

who had stopped or who had changed to pipe had similar mortalities of $9.1 \%$ and $8.8 \%$, respectively, a mortality experience which closely corresponded to the mortality of the $10.2 \%$ among initial non-smokers who remained such. Irrespective of initial smoking status the mortality of those who stopped smoking was similar to that of the non-smokers. Other changes recorded in this Table refer to such small numbers that they are of little importance.

\section{Discussion}

These results confirm that different smoking subgroups of patients with coronary heart disease may have different survival rates. The mortality of all smokers who stopped was similar to that of the nonsmokers, suggesting that the apparent adverse effect of smoking on coronary patients is relatively shortlived.

Cigarette smoking after myocardial infarction was associated with a significantly raised mortality. The mortality of cigar smokers during follow-up was also high and did not differ significantly from the mortality of the cigarette smokers who continued. Those who changed from cigarette smoking to the pipe had a mortality similar to non-smokers but initial pipe smokers who continued had a higher mortality than cigarette smokers who had changed to the pipe. The reason for this difference between the two pipesmoking groups is not clear but the difference was not statistically significant.

We recently reported the carboxyhaemoglobin level of a sample of the original cohort of 664 patients. $^{8}$ Among the 117 subjects in the sample, 13 were pipe smokers and six were cigar smokers. They had mean carboxyhaemoglobin levels of $3 \cdot 2 \%$ and $4 \cdot 1 \%$, respectively, compared with a mean level of $4.3 \%$ for cigarette smokers and $0.56 \%$ for non-smokers or ex-smokers. These findings suggest significant inhalation among pipe and cigar smokers as well as cigarette smokers.
In a recent communication, Wald et al. ${ }^{9}$ reported a higher serum cotinine level in pipe smokers compared with smokers of cigarettes and cigars. Gyntelberg et $a l .1^{10}$ in a study of 5249 apparently healthy men, found heavy cheroot smokers to be at higher risk of fatal and non-fatal myocardial infarction compared with cigarette or pipe smokers. It may be that their findings can be equally applied to patients who have suffered a myocardial infarction.

There was a low mortality recorded among the mixed smoking group of 37 patients. This group included a variety of intermittent and light smokers and cannot be as easily classified as the other smoking groups. The intermittent smokers among this group may at least partially account for their low mortality.

Certain limitations of this study need to be stressed. Apart from the limited number of patients in the pipe and cigar categories we did not quantify cigar smoking initially nor did we quantify pipe and cigar smoking at follow-up. We do have data on carboxyhaemoglobin levels in a sample of 117 drawn from the original cohort of 664 patients.

Further studies are required to clarify the effects of cigar and pipe smoking on prognosis in coronary patients. These studies should include details of quantity smoked and of inhalation. In the meantime our data suggest that, irrespective of initial smoking habits, changing to the pipe may be less harmful than cigar smoking but, in the context to our present limited knowledge, it seems best to advise coronary patients to eschew all forms of smoking.

\section{References}

1 Mulcahy R, Hickey N, Graham I, McKenzie G. Factors influencing long-term prognosis in male patients surviving a first coronary attack. Br Heart $\mathcal{F} 1975 ; 37$ : 158-65.

2 Wilhelmsson C, Vedin JA, Elmfeldt D, Tibblin G, Wilhelmsen L. Smoking and myocardial infarction. Lancet 1975 ; i: 415-20. 
3 Mulcahy R, Hickey N, Graham I, MacAirt J. Factors affecting the 5-year survival rate of men following acute coronary heart disease. Am Heart f 1977; 93: 556-9.

4 Coronary Drug Project Research Group. Cigarette smoking as a risk-factor in men with a prior history of myocardial infarction. F Chronic Dis 1979; 32: 415-25.

5 Salonen JT. Stopping smoking and long-term mortality after acute myocardial infarction. Br Heart $\mathcal{F}$ 1980; 43: 463-9.

6 US Department of Health, Education and Welfare. Smoking and Health. A Report of the Surgeon General, 1979. DHEW Publication No (PHS) 79-500066.

7 Armitage P. Statistical methods in medical research. Oxford: Blackwell, 1971.

8 Ronan G, Ruane P, Graham IM, Hickey N, Mulcahy R.
The reliability of smoking history amongst survivors of myocardial infarction. $\mathrm{Br} \mathcal{F}$ Addict 1981; 76: 425-8.

9 Wald NJ, Idle M, Boreham J, Bailey A, Van Vunakis H. Serum continine levels in pipe smokers: evidence against nicotine as cause of coronary heart disease. Lancet 1981; ii: 775-7.

10 Gyntelberg F, Lauridsen L, Pedersen PB, Schubell K. Smoking and risk of myocardial infarction in Copenhagen men aged 40-59 with special reference to cheroot smoking. Lancet 1981; i: 987-9.

Requests for reprints to Professor Ristéard Mulcahy, Cardiac Department, St Vincent's Hospital, Dublin 4, Republic of Ireland. 